\title{
Simulation studies of the impact of the CMS radiation environment on $\mathrm{RPC}$ detectors
}

\section{Uribe Estrada* ${ }^{a}$, A. Castaneda Hernandez ${ }^{b}$, M. A. Del Rio Viera ${ }^{a}$, S. Carpinteyro Bernardino $^{a}$ \\ on behalf of the CMS collaboration}

${ }^{a}$ Benemerita Universidad Autonoma de Puebla, Puebla, Mexico.

${ }^{b}$ Universidad de Sonora. Apartado Postal: 5-88, Hermosillo, Sonora, Mexico.

E-mail: cecilia.uribe.estrada@cern.ch

\begin{abstract}
The High-Luminosity Large Hadron Collider (HL-LHC) upgrade aims to increase its luminosity by a factor of 5 beyond the LHC's design value, increasing the potential for discoveries after 2025 . The increased collision rate of particles will be a challenge for the CMS systems as higher levels of radiation could degrade them and affect their performance. It is therefore important to understand the expected radiation environment and its impact on the different sub-detectors. In this study we use the FLUKA simulation package to reproduce the radiation environment during CMS Run-2 and the GEANT4 simulation package to estimate its impact on the RPC detectors. Results are compared with measurements collected by the RPC system during 2018 and reasonable agreement is observed. This study serves as a benchmark for future simulations with a Phase-2 (HL-LHC) configuration.
\end{abstract}

Puebla, Mexico

*Speaker. 


\section{The CMS Muon System RPCs}

In the Compact Muon Solenoid (CMS) experiment, muons can be identified and measured individually and accurately. The Resistive Plate Chambers (RPCs) are mainly used as trigger devices, but also contribute to the reconstruction of particles in both barrel and endcap regions up to a pseudorapidity of $|\eta|=1.9$. Background radiation studies play a decisive role in understanding the performance of the detectors, and could help to improve the design for the upgrade of the muon system for the High Luminosity phase [1,2]. The implementation of new RPCs with improved technology (iRPC) at the endcap region will increase the eta coverage up to $|\eta|=2.4$ (Figure 1). The red square indicates where additional RPCs will be placed to extend the muon system coverage [3]. The increase in luminosity during the HL-LHC will increase the potential of discovery but it

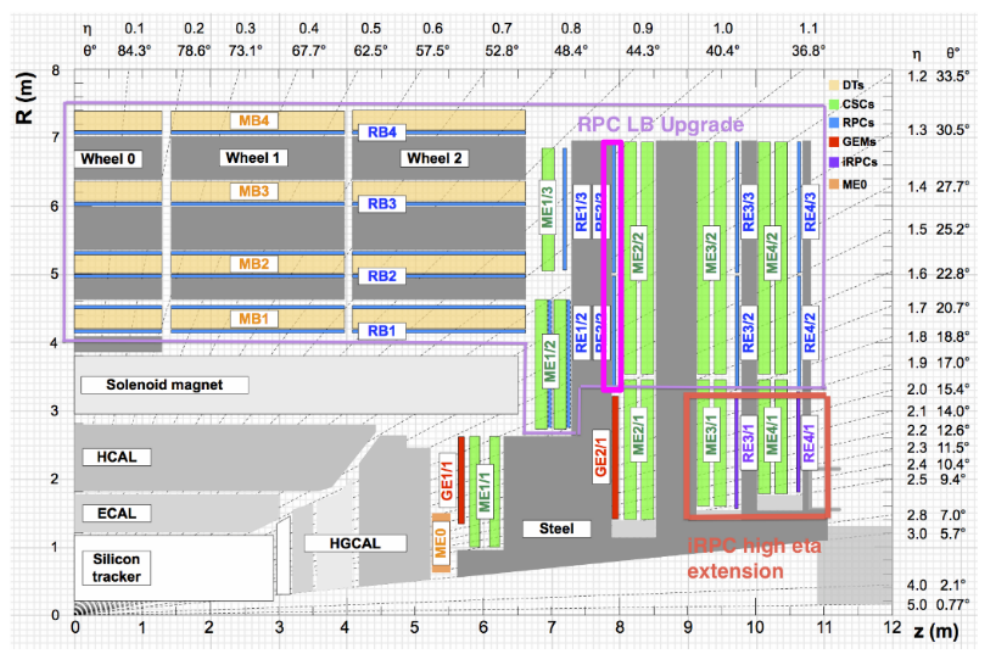

Figure 1: A quadrant of the CMS experiment.

will also increase the radiation background to which the detectors are exposed. Simulation studies help to better understand the parameters which need to be taken into account to keep the detectors safe and performing at high efficiency. The pink rectangle shows the RPC simulation area.

\section{Background Radiation}

Incoming particles at different energies are responsible for different processes in the production of secondary particles in the detector. The expected incident particles' flux has been estimated with a FLUKA simulation taking into account the CMS geometry for Run 2, assuming pp collisions at 13 $\mathrm{TeV}$ at the nominal instantaneous luminosity of $1.5 \times 10^{34} \mathrm{~cm}^{-2} \mathrm{~s}^{-1}$ (Figure 2).

A Monte Carlo (MC) estimation of the flux is shown in Figure 3 as well as the differential distribution of the flux in terms of energy (Figure 4) for $n, \gamma, e^{+}, e^{-}$crossing the volume defined by the 


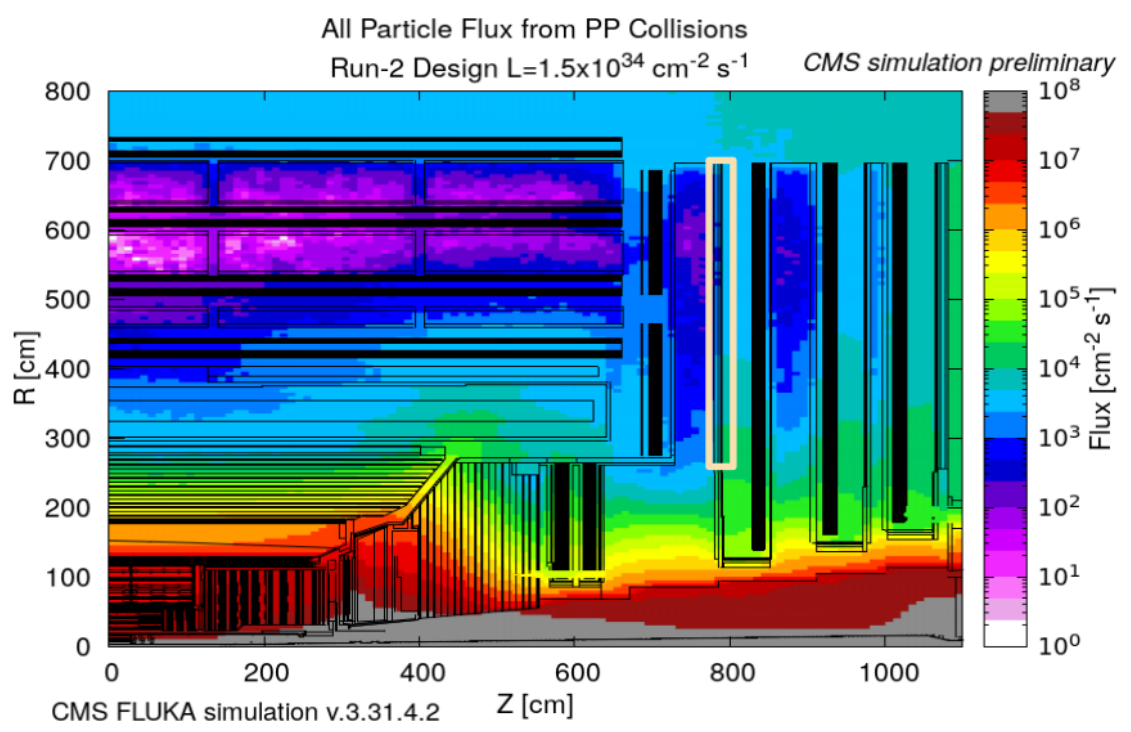

Figure 2: MC simulation of the particle flux in a quadrant of the CMS detector using FLUKA. The particle flux in RE22/23 (shown in the beige rectangle) has contributions in low and high R from leaking between barrel and endcap calorimeter and cavern. In the intermediate part the flux is attenuated due to an effective shielding from other detector elements.

RE22/23 detector stations simulated using FLUKA. In this simulation energy cut-offs for different particles were taken as: hadrons $1 \mathrm{keV}$, muons $1 \mathrm{keV}, n 0.01 \mathrm{MeV}, \gamma 3 \mathrm{keV}$ and $e^{-} 30 \mathrm{keV}$.

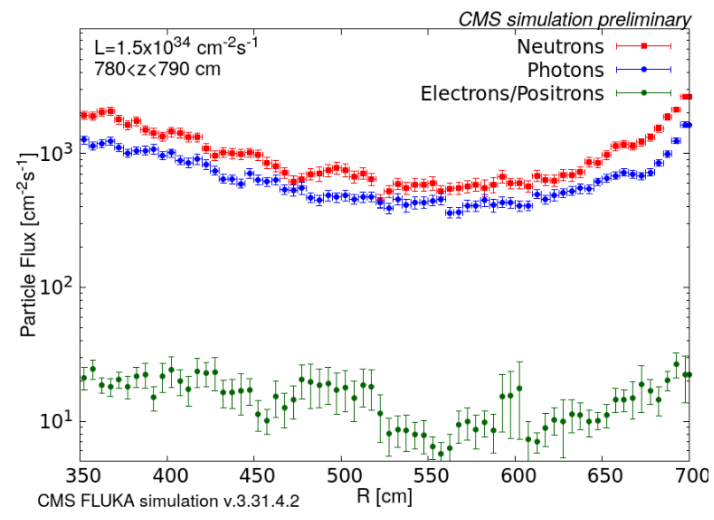

Figure 3: MC estimation of the flux. The inelastic collision cross section used for normalization is 80 $\mathrm{mb}$. The distributions are normalized according to an instantaneous luminosity of $1.5 \times 10^{34} \mathrm{~cm}^{-2} \mathrm{~s}^{-1}$.

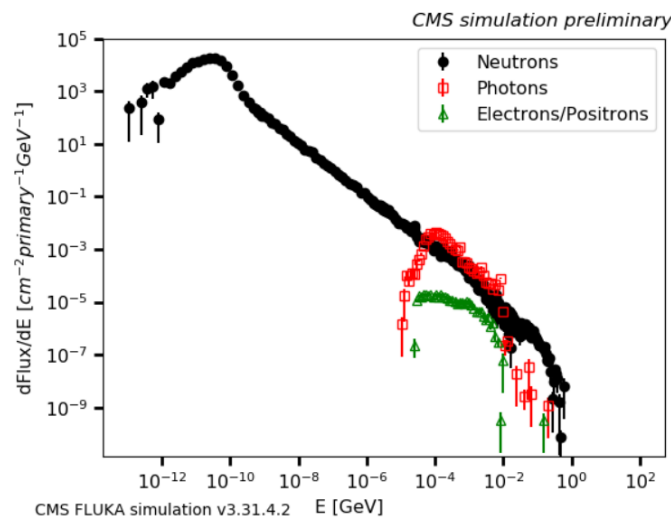

Figure 4: MC differential distribution of flux in energy. The scored energy lower thresholds are $10^{-6} \mathrm{GeV}$ for EM particles and $10^{-14} \mathrm{GeV}$ for neutrons.

\section{Sensitivity vs Energy for RE22}

Sensitivity is defined as the probability for a background particle to create a signal in at least one of the RPC gas gaps. The detailed geometry of the RPC with several layers including the aluminum 
FEB (Front-End Board) cover, PCB FEBs FR4, Mylar sheets, copper cooling panels and pipe (including $\mathrm{H}_{2} \mathrm{O}$ ), honeycomb aluminum panels (including the peripheral and crossbars), Faraday cage, graphite, Bakelite plates, gas gaps, copper strips, etc. was simulated using GEANT4 (Figure $5)$.
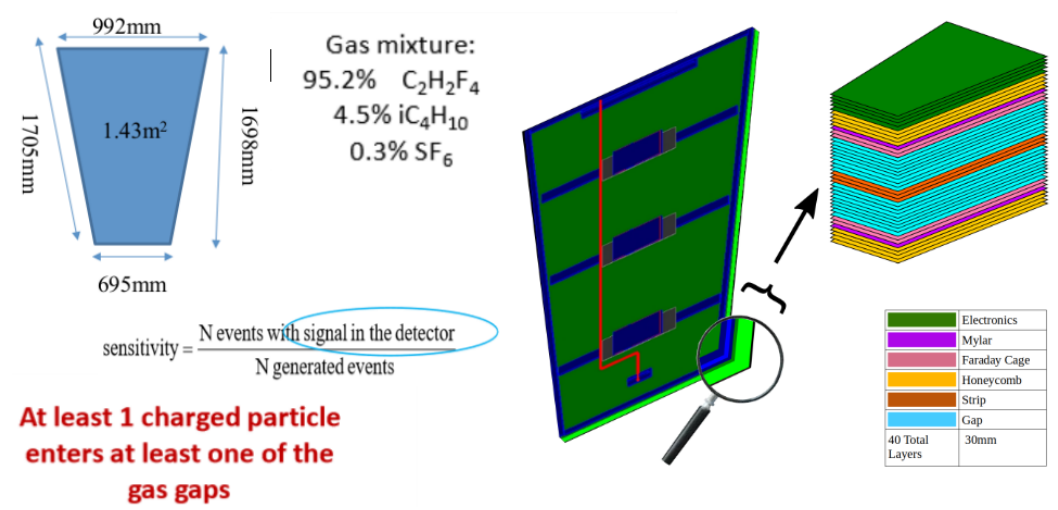

Figure 5: The RE22 geometry used in the GEANT4 simulation. The gas mixture in the RPCs as well as the definition of sensitivity are also given.

Using Monte Carlo simulations we estimate the sensitivity of the double gap RPC detector as a function of the energy of the incident particle for different kinds of particles using GEANT4. The simulation generates 100,000 events per particle: $n, \gamma, e^{+}, e^{-}$, with the resultant sensitivity shown in Figure 6.

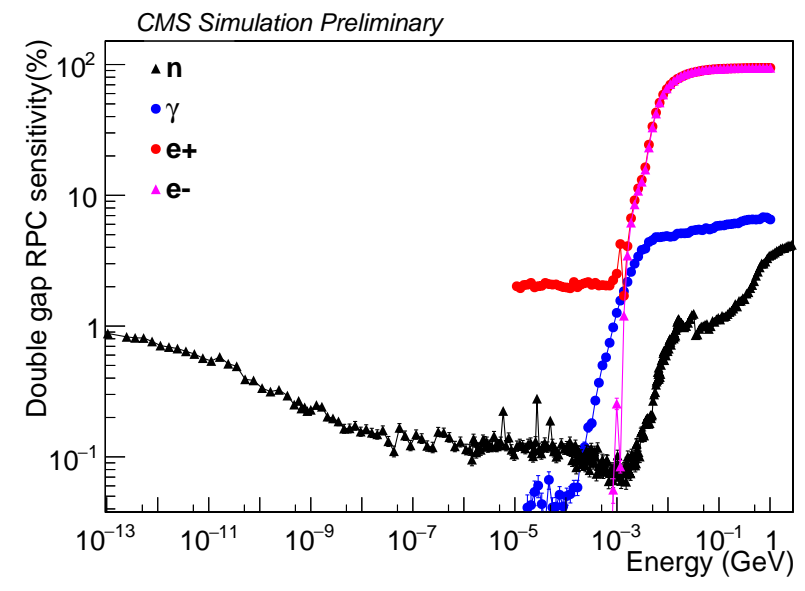

Figure 6: Sensitivity vs energy for a double gap RPC chamber.

\section{Hit Rate}

In order to compare the results obtained from simulation with data we need to convert fluxes to hit rates, where the hit rate is defined as the number of particles reaching the RPC station (particle flux) which is convoluted with a detector response (sensitivity) and is expressed in $\mathrm{Hz} / \mathrm{cm}^{2}$. The expected background hit rate is estimated and presented in Figure 7. 


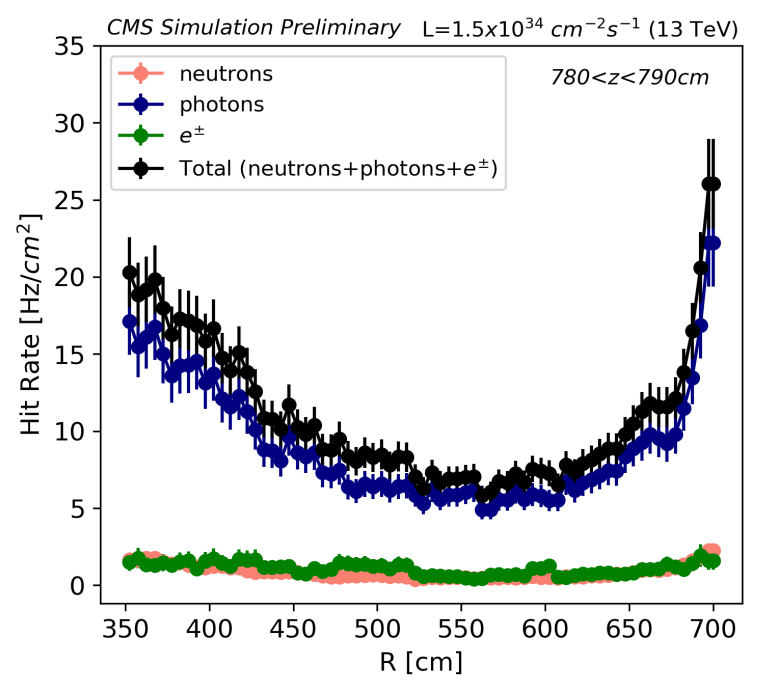

Figure 7: Hit rate MC as a function of the radial distance from the interaction point.

\section{Data vs MC}

The comparison of hit rates as a function of the pseudorapidity between simulation and RPC data collected during 2018 is shown in Figure 8.

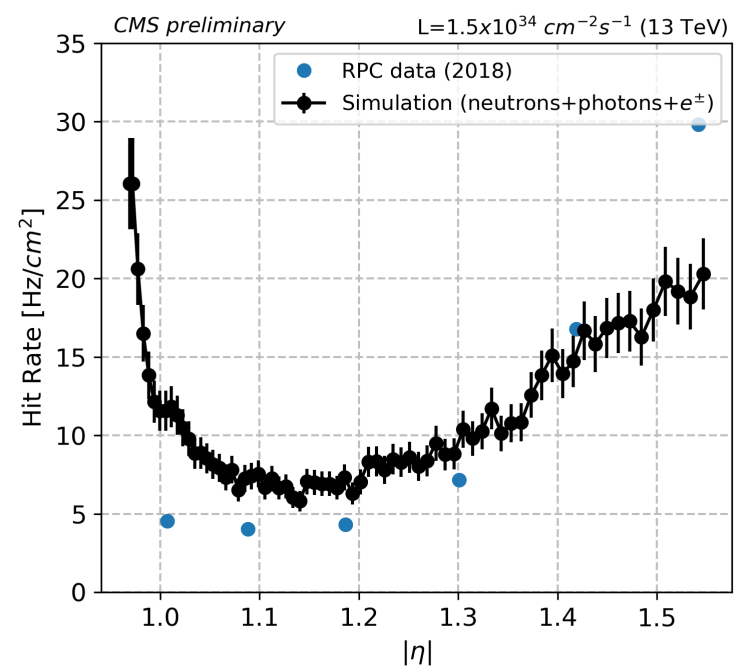

Figure 8: MC and data RPC hit rates as a function of pseudorapidity.

The data and MC show good agreement, with the largest deviation within a factor of two. The RPC hit rate is measured at the strip level during LHC pp collision runs. The strip rate is calculated by using the incremental counts of the RPC trigger link boards. The incremental counts are taken during typical time intervals of the order of $100 \mathrm{~s}$. The resulting rates are then averaged over the 
total runtime and normalized to the strip area. No trigger selection is applied at this stage, resulting in an inclusive measurement of the radiation background rates [4].

\section{Conclusion}

Experimental results corresponding to an instantaneous luminosity of $1.5 \times 10^{34} \mathrm{~cm}^{-2} \mathrm{~s}^{-1}$ were compared to a simulation prediction in which FLUKA and GEANT4 were combined to build a background model. FLUKA was used to derive the particle flux and differential distribution fluence in energy related to the RE22/23 region.

A dedicated GEANT4 simulation was utilized to obtain the response of the RPC to different particles. Results of the hit rate for RE22/23 seem to be consistent with previous estimates. Reasonable agreement (within a factor of 2) is obtained between data and MC, except for low $\eta$ in which several factors could contribute to an increase in particle rate in simulation, such as edge effects, as well as contributions from low energy particles not seen in the detector due to thresholds.

\section{References}

[1] CMS Collaboration, "The Phase-2 Upgrade of the CMS Muon Detectors". CERN-LHCC-2017-012, CMS-TDR-016, Sep. 2017.

[2] C. Uribe Estrada, S. Carpinteyro Bernardino, A. Castaneda Hernandez et al. "RPC Radiation Background Simulations for the High Luminosity Phase in the CMS Experiment". XIV Workshop on Resistive Plate Chambers and Related Detectors, Puerto Vallarta, México, Feb. 2018.

[3] Andrea Gelmi, "improved-RPC for the CMS muon spectrometer upgrade for HL-LHC". Winter LHCC meeting, CERN Geneva (Switzerland). 2019.

[4] S. Costantini et al. [CMS Collaboration], "Radiation background with the CMS RPCs at the LHC", JINST 10 (2015) no.05, C05031. 